\title{
LAS PRODUCCIONES LÍTICAS DEL FINAL DEL EPIPALEOLÍTICO E INICIOS DEL NEOLIITICO EN LA VERTIENTE MEDITERRÁNEA ESPAÑOLA. PROPUESTA METODOLÓGICA
}

\author{
LA PRODUCTION LITHIQUE AU COURS DES PHASES DE L'ÉPIPALÉOLITHIQUE \\ FINAL ET LE DÉBUT DU NÉOLITHIQUE EN ESPAGNE MÉDITERRANÉENNE. \\ UNE PROPOSITION METHODOLOGIQUE
}

ELISA MARÍA DOMÈNECH FAUS $(*)$

\begin{abstract}
RESUMEN
La producción lítica en las fases del Epipaleolítico final y del inicio del Neolítico en el Mediterráneo español está representada por una talla casi exclusiva de lascas, en algunos yacimientos, y de una talla laminar. Para la obtención de lascas se han empleado diferentes métodos: levallois, kombewa y sin predeterminación. En este artículo, se propone también una revisión del concepto "macrolítico" utilizado en los estudios tipológicos y tecnológicos. Los resultados de esta revisión demuestran la existencia de industrias con producciones líticas diferentes.
\end{abstract}

\section{RESUMÉ}

En Espagne méditerranéenne, au cours des phases de l'Épipaléolithique final et le début du Néolithique, la production lithique se caractérise, dans certains gisements, par un débitage presqu'exclusif d'éclats, et dans d'autres sites par un débitage laminaire-lamellaire. Pour l'obtention de ces éclats différentes méthodes ont été employées: levallois, kombewa, sans prédétermination. Nous proposons ici une révision du concept "macrolithique" utilisé dans les études typologiques et technologiques. Les résultats de cette révision mettent en évidence la variabilité des modes de production des industries $l i$ thiques.

(*) Padre Luis Fullana, 10. 03820 Cocentaina. Alicante. Correo electrónico: elisafaus@airtel.net.

El artículo fue remitido en su versión final el 30-III-2000.
Palabras clave: Tecnología. Macrolítico. Lasca. Epipaleolítico. Neolítico.

Mots clés: Espagne méditerranéenne. Technologie. Macrolithique. Éclat. Épipaleolithique. Néolithique.

\section{INTRODUCCIÓN}

En nuestra Tesis Doctoral incluimos (Domènech, 1999) un extenso estudio tecnológico de las industrias líticas de la secuencia epipaleolítica del Abric del Filador (Margalef de Montsant, Tarragona). Los niveles más antiguos ofrecieron una producción lítica preferentemente laminar, en los que variaba la morfología de los soportes en función de su destino final: raspadores, laminitas de dorso, triángulos... Sin embargo, el análisis tecnológico de los niveles más superficiales, sobre todo del Estrato II, mostraba una prioridad por obtener lascas mediante la aplicación de métodos similares a los utilizados en el Paleolítico Medio.

El Estrato II de Filador ha sido datado por cronología relativa en los inicios del Neolítico debido a la aparición de algunos fragmentos de cerámica en una de las cubetas excavadas entre el estrato II y el I (1) (Cebriá et alii, 1981). En otros yacimientos de la vertiente mediterránea occidental también se han podido constatar producciones de lascas (Gar-

(1) Las dataciones absolutas no han sido publicadas en el momento de la redacción de este artículo. 


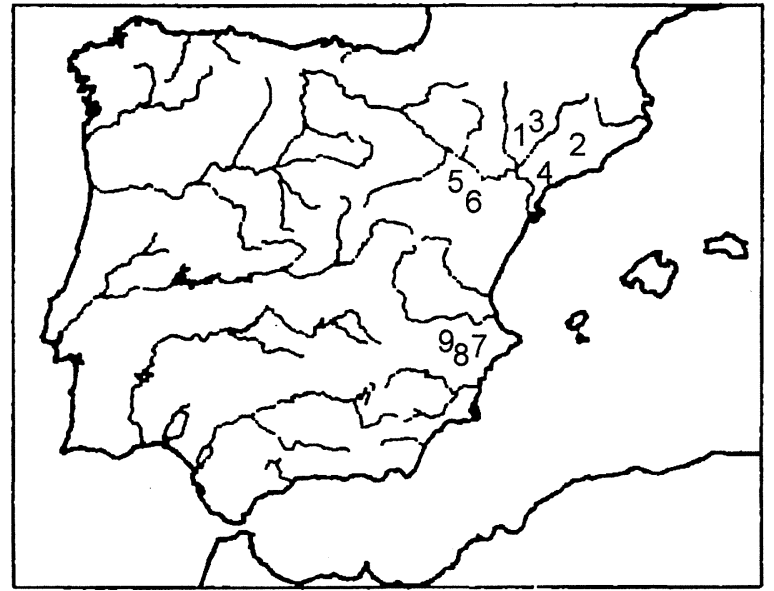

Fig. 1. Mapa de situación de los principales yacimientos citados. 1: Font del Ros (Berga, Barcelona). 2: Roc del Migdia (Vilanova de Sau, Barcelona). 3: Sota Palou (Campdevànol, Barcelona). 4: Filador (Margalef de Montsant, Tarragona). 5: Botiqueria dels Moros (Maella, Zaragoza). 6: Costalena (Maella, Zaragoza). 7: Tossal de la Roca (Vall d'Alcalà, Alicante). 8: Santa Maira (Castell de Castells, Alicante). 9: Barranc de les Calderes (Planes, Alicante).

cía-Argüelles et alii, 1992), aunque en contextos cronológicos más antiguos.

Industrias con un importante componente de lascas y denticulados han sido identificadas entre el VIII y el VII milenio antes del presente en la Font del Ros (Berga, Barcelona) (Terradas, 1995), Roc del Migdia (Vilanova de Sau, Barcelona) (Rodríguez e Yll, 1991), Sota Palou (Campdevànol, Barcelona) (Carbonell et alii, 1985), Botiquería dels Moros, Costalena (Maella, Zaragoza) (Barandiarán y Cava, 1989), el Tossal de la Roca (Vall d'Alcalá, Alicante) (Cacho et alii, 1995), Cova de Santa Maira (Castell de Castells, Alicante) (Domènech, 1991), el Barranc de les Calderes (Planes, Alicante) (Domènech, 1991) (2) (Fig. 1).

No obstante, en la mayor parte de estos yacimientos, la variabilidad tipológica ha desviado el interés del análisis tecnológico hacia el componente geométrico y sus procesos de fabricación (técnica de microburil), elemento muy escaso entre las piezas retocadas del estrato II de Filador. Tal circunstancia no nos ha impedido que intentemos establecer relaciones cronológicas, industriales y culturales entre cada uno de estos yacimientos a partir

(2) Se ha realizado una selección de yacimientos de cuyo estudio pudiera extraerse la información necesaria para ser comparados con el estrato II de Filador, ya que las distintas metodologías utilizadas no permiten una lectura fácil de sus datos.

\begin{tabular}{|c|c|c|c|}
\hline YACIMIENTO & \begin{tabular}{|l|} 
UNIDAD \\
ESTRATIGRÁFICA
\end{tabular} & \begin{tabular}{|l} 
ASIGNACIÓN \\
CULTURAL
\end{tabular} & $\begin{array}{l}\text { DATACIONES } \\
\text { ABSOLUTAS }\end{array}$ \\
\hline FONT DEL ROS & SG & Mesolítico & $8150 \pm 590$ B.P. \\
\hline ROC DEL MIGDIA & $\begin{array}{lll}\text { Perfil } & 9 / 10 & \text { (desde } \\
\text { oeste) } & & \\
\end{array}$ & Epipaleolítico & $\begin{array}{l}7280 \pm 370 \text { B.P. } \\
7950 \pm 370 \text { B.P. } \\
8190 \pm 320 \text { B.P. }\end{array}$ \\
\hline SOTA PALOU & Único nivel & Mesolítico & $\begin{array}{l}8540 \pm 180 \text { B.P. } \\
9060 \pm 380 \text { B.P. }\end{array}$ \\
\hline $\begin{array}{l}\text { BOTIQUERIA } \\
\text { DELS MOROS }\end{array}$ & NIVEL 2 & $\begin{array}{l}\text { Epipaleolítico con } \\
\text { geométricos }\end{array}$ & $7550 \pm 200$ B.P. \\
\hline COSTALENA & NIVEL c3 & $\begin{array}{l}\text { Epipaleolítico con } \\
\text { geométricos }\end{array}$ & $6420 \pm 250$ B.P. \\
\hline \multirow{3}{*}{$\begin{array}{lll}\text { TOSSAL } & \text { DE } & \text { LA } \\
\text { ROCA } & & \end{array}$} & NIVEL I & $\begin{array}{l}\text { Epipaleolítico con } \\
\text { geométricos }\end{array}$ & $\begin{array}{l}7660 \pm 80 \text { B.P. } \\
7560 \pm 80 \text { B.P. }\end{array}$ \\
\hline & NIVEL IIa & $\begin{array}{l}\text { Epipaleolítico con } \\
\text { geométricos }\end{array}$ & $\begin{array}{l}8350 \pm 120 \text { B.P. } \\
8050 \pm 90 \text { B.P. }\end{array}$ \\
\hline & NIVEL IIb & $\begin{array}{l}\text { Epipaleolitico con } \\
\text { geométricos }\end{array}$ & $\begin{array}{l}9150 \pm 100 \text { B.P. } \\
8530 \pm 90 \text { B.P. }\end{array}$ \\
\hline $\begin{array}{l}\text { COVA DE SANTA } \\
\text { MAIRA }\end{array}$ & NIVEL III & Epipaleolitico & $\begin{array}{l}\text { Sin dataciones } \\
\text { absolutas }\end{array}$ \\
\hline $\begin{array}{l}\text { BARRANC DE LES } \\
\text { CALDERES }\end{array}$ & NIVEL I Y II & Epipaleolitico & $\begin{array}{l}\text { Sin dataciones } \\
\text { absolutas }\end{array}$ \\
\hline
\end{tabular}

Tab. 1. Unidades estratigráficas, asignación cultural y dataciones absolutas de los yacimientos mencionados.

de los datos publicados y de las reproducciones gráficas (3) (Tab. 1).

\section{PROPUESTA METODOLÓGICA PARA EL ESTUDIO DEL MATERIAL LÍTICO TALLADO DEL ESTRATO II DE FILADOR}

Los principios metodológicos que hemos seguido están basados en el concepto de Cadena operativa establecido en la década de los años sesenta por A. Leroi-Gourhan (1964); mientras que para su aplicación práctica se han seguido los trabajos de J. Tixier (1991), E. Boëda (1994) y J. Pelegrin (1995).

En la reconstrucción de las cadenas operativas de los procesos de talla del estrato II de Filador han sido identificados dos tipos de producciones: de lascas y laminar. La presentación de ambas se hará por separado ya que corresponden a procesos de troceado diferentes, para finalmente poder cotejarlas según las distintas gestiones de la materia prima, las técnicas de talla y la fabricación de soportes (Domènech, 1998).

(3) Las dataciones absolutas utilizadas son las publicadas en el momento de redacción del artículo. 


\section{Análisis tecnológico}

Producción de lascas

Dentro de la producción de lascas se han seguido dos procesos de obtención de soportes distintos. Estos son:

a. Producción de grandes lascas corticales extraídas de bloques de sílex no conformados previamente para, posteriormente, utilizarlas directamente, dejando huellas de uso, o transformandolas en forma de retoques sobreelevados/profundos con delineación denticulada (4). Para la fabricación de estas grandes lascas se emplearon bloques de gran tamaño, con los suficientes planos naturales como para ser explotados desde diferentes ángulos.

Entre el conjunto lítico recuperado se documentó un lote importante de pequeñas lasquitas, algunas de ellas clasificadas como janus -retoque de la cara de lascado-, resultantes de la fabricación de este tipo de denticulados. Según la experimentación que realizamos para la clasificación de estas piezas, se extraería un número importante de pequeñas lascas para dar mayor robustez al filo.

b. Obtención de soportes anchos mediante la explotación de una superficie siguiendo el principio del método levallois (Boëda, 1994). La producción resultante ha ofrecido una amplia gama de soportes anchos y cortos extraídos de superficies ligeramente acondicionadas. Dentro de este proceso podrían incluirse algunas lascas janus-kombewa, procedentes del aprovechamiento de la cara inferior de una lasca. En este último caso, no siempre la cara de lascado presenta la regularidad suficiente para ser explotada de forma directa, de ahí que se requiera el acondicionamiento periférico, además de la preparación de un plano de percusión.

El estudio traceológico ha demostrado la utilización de los soportes brutos sin retocar, en especial de algunas lascas janus-kombewa. El resto de los productos de plena talla presentan pequeños retoques marginales o frentes de raspador...

\section{Producción de láminas}

Entre la producción lítica del estrato II de Filador contamos con testimonios significativos de la talla laminar. Tanto los núcleos como los restos de preparación/regularización y los propios productos

(4) Retoque "estilo campiñoide". de plena talla han permitido reconstruir procesos de talla laminares. Por otra parte, a partir de los datos que poseemos no se puede hablar de una talla laminar en un sentido clásico, dada la escasa presencia de núcleos laminares. Pero, la diversidad productiva ofrecida por el análisis tipométrico y morfológico muestra una amplia variedad de procesos aplicados.

Tipométricamente se distinguieron los siguientes tipos:

A. 25-20 mm de longitud/ $10-5 \mathrm{~mm}$ de amplitud. B. 25-35 mm de longitud/ $17-10 \mathrm{~mm}$ de amplitud. C. $45-65 \mathrm{~mm}$ de longitud/ $30-25 \mathrm{~mm}$ de amplitud. D. $65-45 \mathrm{~mm}$ de longitud/30-25 mm de amplitud.

Contrariamente a los datos ofrecidos por la producción de lascas, los soportes laminares ofrecen una mayor variedad tipológica con la presencia de microburiles, triángulos y fracturas retocadas. Tal variedad demuestra la aplicación de diversos métodos de talla laminar, a pesar de que no hayan podido ser reconstruidas todas sus cadenas operativas en el yacimiento (Fig. 2).

\section{LA PRODUCCIÓN INTENCIONAL DE LASCAS EN OTROS YACIMIENTOS EPIPALEOLÍTICOS Y NEOLÍTICOS DE LA VERTIENTE MEDITERRÁNEA ESPAÑOLA}

Desafortunadamente no contamos con estudios tecnológicos publicados o, si existen, la metodología utilizada es distinta a la nuestra. Sin embargo, a través de la adaptación de los distintos métodos de análisis y de las reproducciones gráficas, hemos podido observar producciones de lascas en distintos yacimientos del final del Epipaleolítico/Neolítico en la vertiente mediterránea española (5).

\section{Cataluña}

De las latitudes más septentrionales contamos con tres yacimientos emblemáticos en los que se ha identificado una industria con un importante porcentaje de piezas definidas como "macrolíticas" y/ o denticuladas.

(5) Somos conscientes de que el número de ejemplares que mostramos es incompleto, ya que conocemos la existencia de otros yacimientos con las mismas características en fase de excavación o de estudio. 

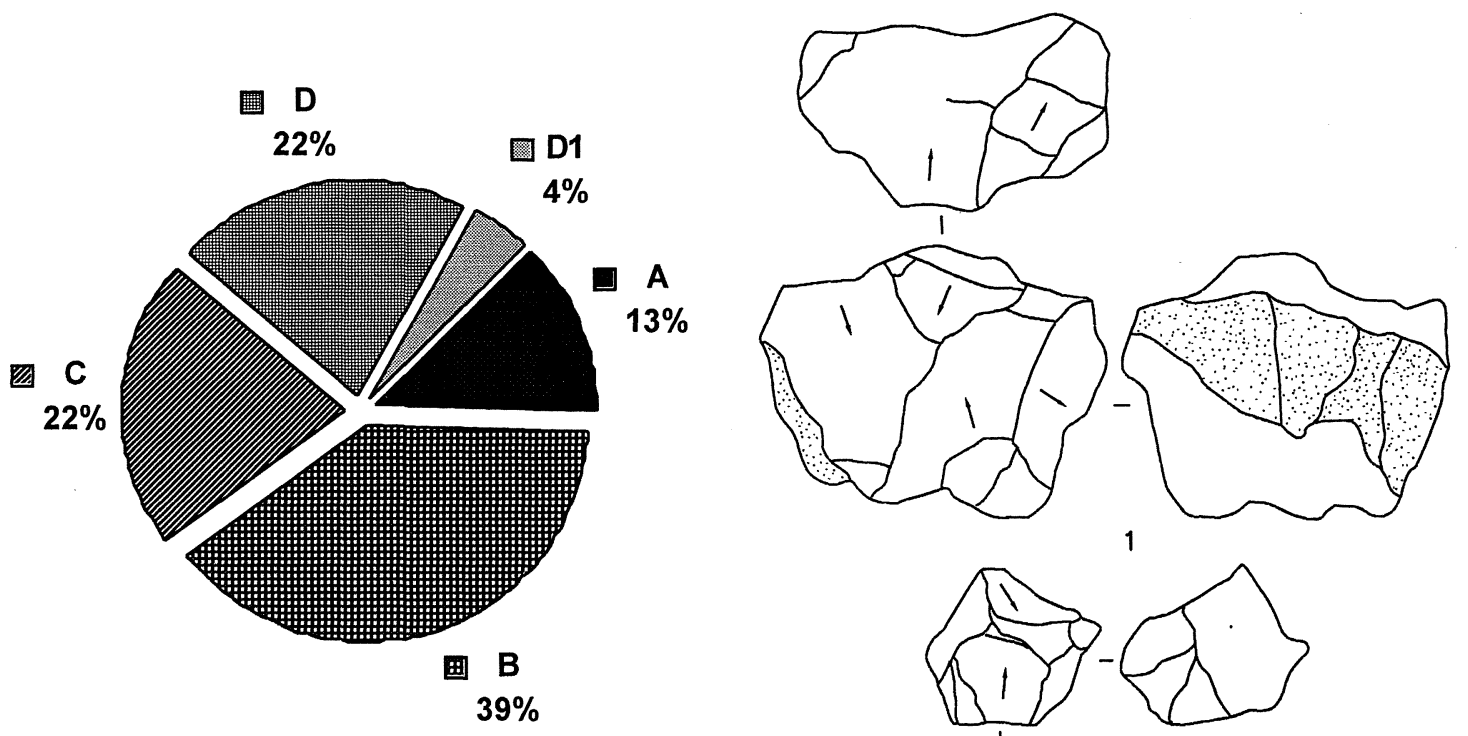

1

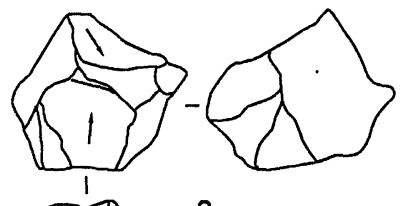

2

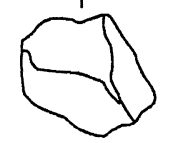

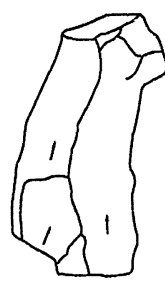

3
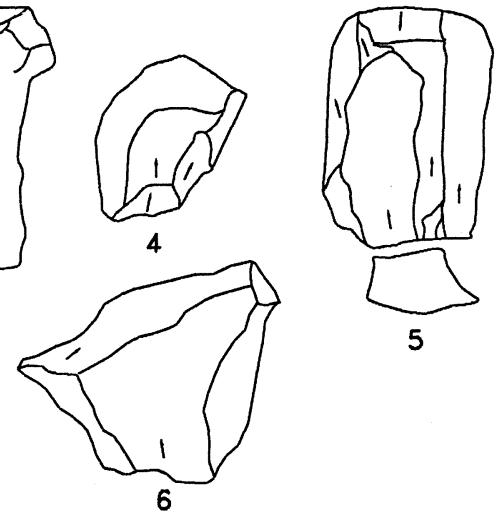

5

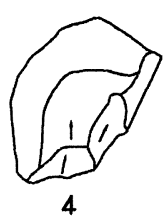

4

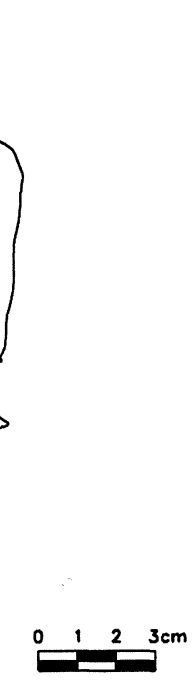

(2)
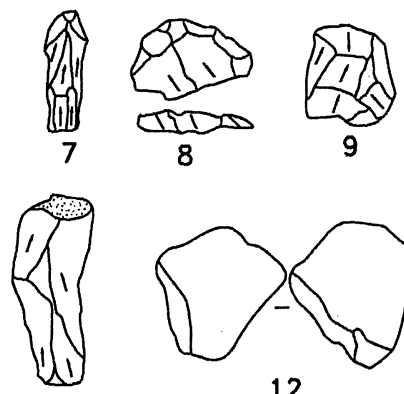

11

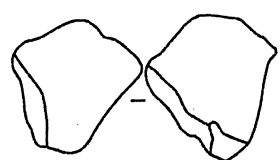

12
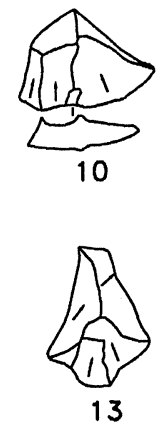

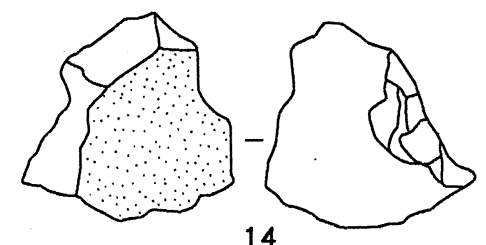

14
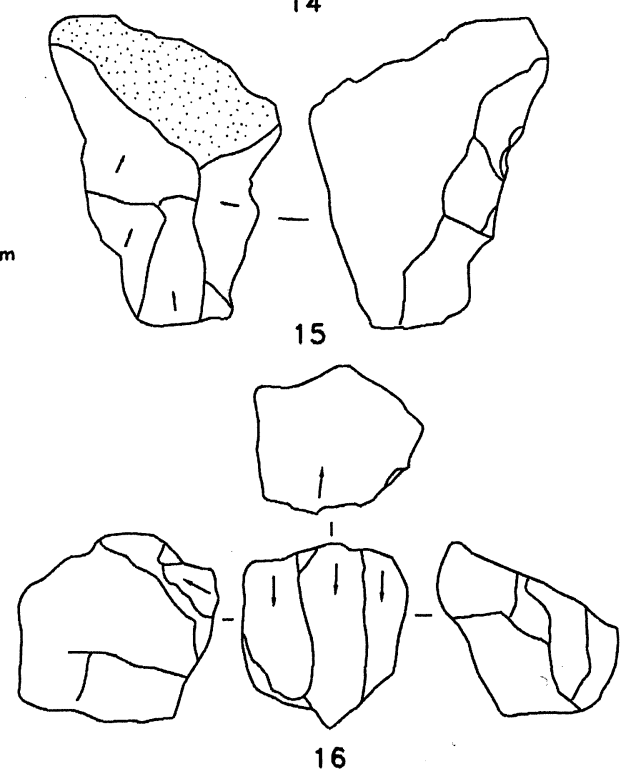

16

Fig. 2. Gráfico Estrato II de Filador (Margalef de Montsant), Producción laminar. A: 25-20 mm de longitud/10-5 mm de amplitud. B: 25-35 mm de longitud/17-10 mm de amplitud. C: 45-65 mm de longitud/30-25 mm de amplitud. D: 65-45 mm de longitud/30-25 mm de amplitud. 1: Núcleo de grandes lascas. 2: Núcleo de preparación de una superficie o explotado mediante el "método levallois" para la talla de lascas. 4 y 12: Lascas extraídas de la cara bulbar preparada de una lasca. 6 , 8, 9, 10, 13: Lascas extraídas de un núcleo preparado por el "método levallois". 3, 5, 7, 11: Talla laminar. 14: Pieza denticulada con retoque simple/sobreelevado profundo inverso. 15: Denticulado. 16: Núcleo de laminitas. 


\section{a. Sota Palou (Campdevànol, Barcelona)}

El estudio tecnológico y petrológico muestra un uso diferencial de las distintas materias primas. El sílex y la lidita, dada su aptitud ante la talla, son buscados para la producción laminar, entre las que se comprueba la existencia de una mayor diversidad tipológica, mientras que del cuarzo sólo se obtienen lascas cuyos soportes retocados han sido clasificados como denticulados (Carbonell et alii, 1985).

\section{b. La Font del Ros (Berga, Barcelona)}

Las materias primas talladas o destinadas a ser transformadas por el retoque son principalmente el sílex, el cuarzo y la cuarcita. En este yacimiento la talla laminar ha debido combinarse con una producción de lascas, coincidente con la explotación centrípeta de algunos de sus núcleos o la producción de grandes lascas a partir de bloques de cuarzo o de cuarcita (Terradas, 1995).

\section{c. Roc del Migdia (Vilanova de Sau, Barcelona)}

Los niveles epipaleolíticos de este yacimiento ofrecen un interesante esquema operativo de talla de lascas en caliza. El sistema de explotación tipo Quina establecido por A. Turq se convierte ahora en un método de talla apropiado para una materia como la caliza, de calidad inferior al sílex, cuyo único objetivo es obtener lascas o soportes "poco definidos". En cambio el sílex, escaso, está representado por soportes laminares y piezas retocadas con una ausencia casi exclusiva de núcleos y restos de talla (Rodríguez e Yll, 1991).

\section{Aragón}

A pesar de que esta región no se encuentre en línea directa de la costa mediterránea, su Prehistoria Antigua siempre ha presentado elementos comunes con el litoral mediterráneo. Dos yacimientos emblemáticos, Botiqueria dels Moros y Costalena, han sido referencia obligada para las investigaciones sobre el final del Epipaleolítico-Neolítico para el País Valenciano (Juan, 1990, 1992). Por otra parte, de estos yacimientos no se ha publicado ningún estudio tecnológico en el que se reconozca una producción de lascas similar a la de Filador.
En el estudio "tecnotipológico" del Abrigo de Costalena se hacía una revisión del término "macrolítico", en el que se diferenciaban dos categorías "morfo-tecnológicas" distintas. Una representada por piezas sobre cantos rodados y otra de piezas sobre lascas gruesas denticuladas con retoque "estilo campiñoide", entendiéndose como tal "algunos utensilios trabajados sobre lascas altas mediante retoque profundo algo tosco (bifacial a menudo, o sólo inverso) que las conforman en piezas gruesas de bordes ligeramente denticulados o con amplias muescas, cuyas huellas de retoque pueden presentar estigmas de astillamiento algo similares a lo escaleriforme" (Barandiarán y Cava, 1989: 105).

Conjuntamente a estas piezas aparecen los trapecios y triángulos fabricados a partir de la técnica del microburil, así como raspadores, laminitas de dorso, talla laminar... No obstante, nos interesa poder identificar entre todos estos objetos líticos una talla intencional de lascas. Así, a lo largo de toda la secuencia de Costalena, aparte de los denticulados con retoque estilo campiñoide, advertimos la presencia de lascas y núcleos resultantes de la aplicación del método levallois (6).

\section{País Valenciano}

En tres yacimientos con niveles datables entre el final del Epipaleolítico e inicios del Neolítico se ha contabilizado un buen número de piezas con muescas y denticulados: Tossal de la Roca, Cova de Santa Maira y Barranc de les Calderes. Pero no en todos ellos se ha contado con el mismo registro arqueológico, mientras que en unos los problemas postdeposicionales han hecho difícil la ubicación cronológica de sus niveles, en otros se cuenta con un estudio interdisciplinar bastante completo.

\section{a. Tossal de la Roca (Vall d'Alcalà, Alicante)}

Este yacimiento ha ofrecido una secuencia ininterrumpida bastante dilatada que arranca desde niveles con materiales magdalenienses y finaliza en los inicios del Neolítico (Cacho, 1990).

(6) Nos basamos en las representaciones gráficas de la monografía de Costalena. Véanse las figuras $12\left(n^{\circ} 2\right.$ y 3$)$ y $16\left(n^{\circ} 1,2\right.$ y 4) (Barandiarán y Cava, 1989). 


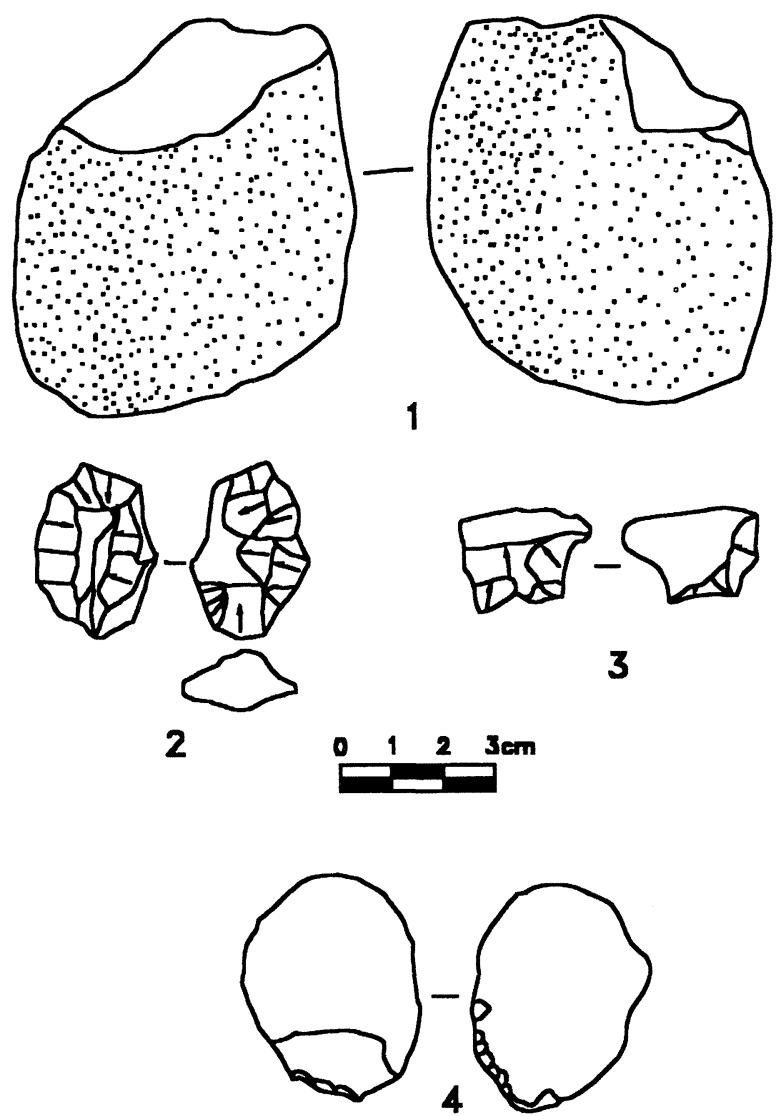

Fig. 3. Santa María (Castell de Castells, Alicante), nivel III. 1: Pieza macrolítica, canto tallado de caliza. 2: Núcleo preparado mediante el "método levallois". 3: Lasca "levallois". 4: Lasca "kombewa".

El elemento "macrolítico" aparece con mayor frecuencia a partir del nivel IIb del sector exterior ubicable secuencialmente en un Epipaleolítico medio. Su presencia junto con piezas denticuladas con retoque sobrelevado-simple/profundo es la única diferencia existente respecto al nivel más antiguo. Este elemento se irá incrementando en los niveles más recientes, alcanzando su apogeo en el nivel I del sector exterior, conjuntamente con los trapecios y los microburiles.

El estudio tecnológico no ha sido realizado por el momento, de ahí que tan sólo nos podamos basar en las anotaciones y dibujos de las diversas publicaciones. Así, a pesar de que no se haya representado ningún núcleo, se visualizan grandes lascas denticuladas, lascas janus-kombewa y, sobre todo, piezas con retoque sobreelevado-simple/ profundo (Cacho et alii, 1995, véanse figuras 36, $38,39$ y 40$)$.

T. P., 57, n. ${ }^{\circ} 1,2000$

\section{b. Cova de Santa Maira (Castell de Castells, Alicante)}

De este yacimiento se han publicado los resultados preliminares de un amplio sondeo (estudio del material arqueológico). Su secuencia cronocultural abarca desde el Epipaleolítico microlaminar o antiguo hasta el Neolítico con ciertos vacíos secuenciales debidos a los problemas deposicionales que anteriormente mencionabamos. Así, el nivel III, poseedor de los denticulados y de "las piezas macrolíticas", aparece surcado por madrigueras de animales, las cuales han debido contaminar los niveles de contacto entre el Epipaleolítico antiguo y el Neolítico, aunque su posición estratigráfica permite que sea datado por cronología relativa entre ambas fases (Domènech, 1991, 1995).

No obstante, en este conjunto lítico hemos identificado claramente una producción intencional de lascas a través de los núcleos y de los restos de ta1la. En el nivel III de Santa Maira existen núcleos en los que se ha aplicado el método levallois, lascas resultantes de tal método, piezas macrolíticas en sílex, piezas con retoque sobreelevado/profundo y algún elemento geométrico (tranchet) (Fig. 3).

\section{c. Barranc de les Calderes (Planes, Alicante)}

La sedimentación de este gran abrigo posiblemente corresponda al momento de formación de las terrazas holocénicas del mismo barranco. En el sondeo practicado se diferenciaron siete niveles, pero tan sólo dos tenían material arqueológico de interés, niveles I y II. A pesar que el nivel más reciente ofreció varios fragmentos de cerámica neolítica la estructura industrial apenas difiere respecto al infrapuesto (Domènech 1991, 1995).

El componente tipológico de este yacimiento es prácticamente idéntico al del estrato II de Filador, en el que las muescas y denticulados, así como las piezas macrolíticas tienen el dominio casi exclusivo de las piezas retocadas. De igual forma, desde el punto de vista tecnológico, asistimos a una intención productiva de lascas, de la que se han documentado núcleos de explotación en superficie o levallois, lascas, denticulados con retoque sobreelevado/profundo, grandes piezas denticuladas, en contraste con otras piezas microlíticas resultado de la fabricación de los denticulados (Fig. 4). 


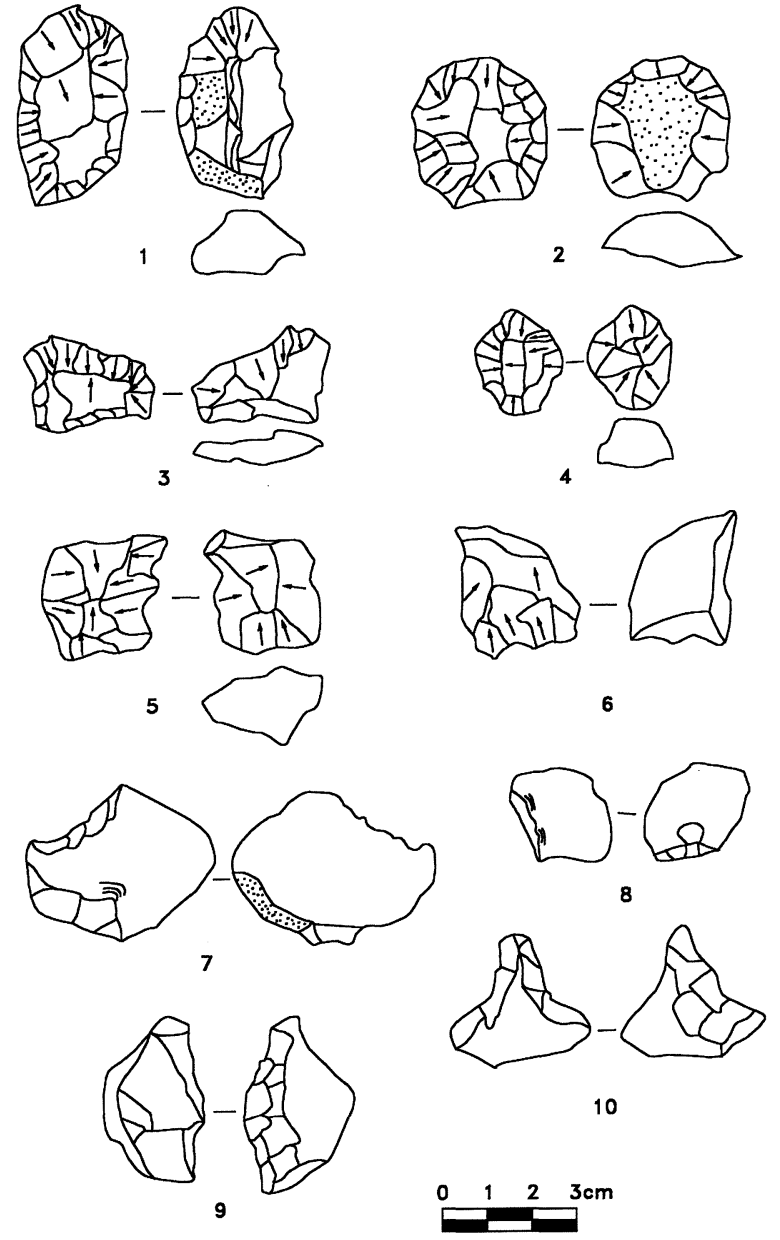

Fig. 4. Barranc de les Calderes.1, 2, 3, 4 y 5: Núcleos preparados por el "método levallois" agotados. 6: Lascas levallois. 7 y 8: Lascas kombewa. 9 y 10: Lascas con retoque simple/sobreelevado-profundo o "estilo campiñoide".

\section{EL UTILLAJE "MACROLÍTICO" Y LA PRODUCCIÓN DE LASCAS EN LAS INDUSTRIAS MEDITERRÁNEAS ESPAÑOLAS DEL FINAL DEL EPIPALEOLÍTICO Y PRINCIPIOS DEL NEOLÍTICO}

Antes de poder interpretar los datos aquí presentados, es conveniente definir el término "macrolítico" tan utilizado en la descripción de las industrias de los yacimientos que acabamos de exponer. Esa denominación en numerosas publicaciones se ha prestado a confusión, al ser definidos dentro de este tipo genérico objetos líticos pertenecientes a procesos productivos muy distintos.

Para I. Barandiarán y A. Cava (1989) el término "macrolítico" puede aplicarse a utensilios fabricados sobre cantos rodados, mientras que el resto de los denticulados que hemos descrito en algunos yacimientos, sólo presentan un rasgo común: el retoque simple-sobreelevado/profundo. Sin embargo, para estos autores existen otras características "tipotecnológicas" que permitirían establecer tipos distintos como el pico entre muescas, las piezas estranguladas...

Tales hechos nos inducen a pensar que las piezas macrolíticas sobre caliza de los tres yacimientos catalanes -Sota Palou, la Font del Ros, Roc del Migdia- podrían incluirse dentro del grupo "macrolítico". En cambio, los denticulados con retoque simple sobreelevado/profundo englobarían otro grupo de piezas y formarían parte de procesos e intenciones de talla distintos.

A partir de los datos expuestos y de la redefinición del término "macrolítico", se pueden describir varias cadenás operativas destinadas a la producción de lascas desde el punto de vista tecnológico y cronocultural.

Las distintas interpretaciones tecnológicas que se han hecho permiten inducir la existencia de tres procesos distintos en la obtención de soportes anchos y cortos (lascas):

- Explotación en superficie de un soporte nuclear (método levallois). Este tipo de explotación requiere de la preparación de una superficie de planos a partir de los cuales extraer la lasca directamente (en el caso de las caras superiores de una lasca) o para el acondicionamiento de la superficie de talla. Los núcleos pueden encontrarse en la Font del Ros (7), en el estrato II de Filador, el Tossal de la Roca, Botiqueria dels Moros, Costalena, Cova de Santa Maira y el abric del Barranc de les Calderes.

- Extracción de grandes lascas de forma aleatoria. Los soportes obtenidos serán destinados para la fabricación de las grandes piezas denticuladas, hecho constatado en las industrias de los principales yacimientos considerados.

- Extracción de lascas por el método kombe$w a$. Este método ha podido ser comprobado en el estrato II de Filador, Botiqueria dels Moros, Costalena, Tossal de la Roca y el Barranc de les Calderes.

(7) En nuestra tesis doctoral pusimos en duda la interpretación que se hizo de la descripción de las distintas cadenas operativas, de ahí que ahora tomemos los datos aquí expuestos con reserva ya que puede tratarse de preformas de núcleos laminares (Domènech, 1999). 


\section{DISCUSIÓN}

La falta de trabajos tecnológicos más generalizado, así como la precaria información que poseemos de yacimientos como el Barranc de les Calderes o de otros, ahora posiblemente en fase de excavación/estudio, no permite sacar conclusiones definitivas. De ahí que en este artículo se haya pretendido mostrar los resultados obtenidos con la actualización hecha de algunas de las industrias del Epipaleolítico Final y Neolítico, revisión que igualmente puede ser aplicada a otros conjuntos industriales contemporáneos. Por tanto, ante tales hechos, nos limitamos a reflexionar sobre términos como "macrolítico" o denticulados, la asimilación método levallois al Musteriense o la identificación del Epipaleolítico Final con geométricos...

La definición "morfotipológica" de los denticulados con retoque simple-sobreelevado/profundo o con retoque "estilo campiñoide" queda claramente representada en casi todos los yacimientos a excepción de Sota Palou, Roc del Migdia y la Font del Ros (8). En estos casos se trata de denticulados cuyo proceso de fabricación difiere del que podríamos encontrar en otros contextos del Epipaleolítico antiguo o incluso del Paleolítico Superior.

Sin embargo, el hecho de que hayamos identificado un método de talla ya aplicado durante el Paleolítico Medio -método levallois-, no significa que los productos resultantes tengan el mismo destino o funcionalidad que las industrias musterienses -aunque por el momento carezcamos de estudios traceológicos generalizados de ambos periodos-. Por tanto, podemos afirmar que los métodos y las técnicas de talla no pueden ser representativos de procesos evolutivos o culturales determinados, ya que según las necesidades pueden resurgir estrategias o métodos de talla propios de momentos anteriores.

El significado cultural y cronológico de este tipo de producciones rompe con el tradicional esquema que se había presentado hasta el momento para la secuencia epipaleolítica, en el que se diferenciaban dos grandes complejos líticos, microlaminar y geométrico (Fortea, 1973). Los conjuntos líticos que no presentaban los elementos característicos de estos complejos eran considerados como casos especiales o, incluso, recientemente, se han pretendi-

(8) Ausencia debida a las distintas metodologías utilizadas en la clasificación de estas piezas. do crear nuevas facies culturales, las cuales, por su posición estratigráfica, podrían situarse entre el Epipaleolítico antiguo y el reciente o geométrico, de "clara tendencia sauveterriense" (Aura y Pérez, 1992).

Además, como hemos podido comprobar a lo largo de este trabajo, no en todos los yacimientos en los que se ha definido el término "macrolítico" se puede hablar de procesos idénticos de producción lítica. En el caso de Sota Palou o Font del Ros, junto a una producción laminar existe una talla de cantos de caliza -"piezas macrolíticas"- o en otras materias primas, dada la escasez de sílex, a los que posiblemente se acompañe la producción de otras piezas geométricas.

No obstante, en el resto de los yacimientos se repite una presencia bastante evidente de las piezas con retoque simple/profundo y de la producción de lascas a partir del final del Epipaleolítico antiguo o microlaminar, cuya perduración llegará hasta los inicios del Neolítico. Un ejemplo claro de tal perduración podría ser el Tossal de la Roca, donde, desde el nivel IIb hasta el Neolítico, la constatación de este tipo de piezas va en aumento conjuntamente con trapecios fabricados mediante la técnica del microburil (9).

De igual forma, tal esquema se repite en Botiqueria dels Moros y Costalena, sobre todo, en el último yacimiento que, a partir de un "nivel d genérico", la producción intencional de lascas y la fabricación de piezas con retoque "estilo campiñoide" convivirá hasta bien avanzado el Neolítico con la talla laminar, los trapecios y triángulos, laminitas de dorso...

Por tanto, a partir de estas reflexiones, no resulta aventurado pensar que pueda existir una cierta especialización en distintos asentamientos a finales del Epipaleolítico (10), en el que sólo aparezcan los denticulados o se necesite prioritariamente producir lascas, en tanto que los otros elementos industriales característicos de estas fases -geométricostengan una presencia testimonial. En este caso se incluiría al estrato II de Filador, el Barranc de les Calderes o el nivel III de Santa Maira -cuya cronología relativa podría oscilar entre el final del Epipa-

(9) Sería interesante verificar esta secuencia en la Cueva de la Cocina, pero ante la falta de un estudio tecnológico, así como de la publicación de otros datos, dejamos para otra ocasión el poder incluir este yacimiento entre nuestras valoraciones cronoculturales.

(10) Cambios o especializaciones ya mencionadas por otros autores (Barandiarán y Cava, 1992; Aura y Pérez, 1992) en otras ocasiones y que aquí venimos a confirmar.

T. P., 57, n. $^{\circ} 1,2000$ 
leolítico microlaminar y el Neolítico-, dentro de un sistema general de explotación de recursos similar al que podría haber tenido Cocina, el Tossal de la Roca, los yacimientos bajoarogoneses... en fases cronológicamente contemporáneas.

\section{BIBLIOGRAFÍA}

Aura, J.E. y Pérez, M. (1992): “Tardiglaciar y Postglaciar en la región mediterránea de la Península Ibérica (13500-8500 B.P.): transformaciones culturales y económicas". Saguntum, 25: 25-47

BARANDIARÁN, I. (1978): "El abrigo de la Botiqueria dels Moros. Mazaleón (Teruel). Excavaciones arqueológicas de 1974". Cuadernos de Prehistoria y Arqueología Castellonense, 5: 49-138

BARANDIARÁN, I. y CAVA, A. (1989): La ocupación prehistórica del abrigo de Costalena (Maella, Zaragoza). Colección Arqueología y Paleontología, 6, Serie ArqueologíaAragonesa, Monografías. Diputación deAragón. Zaragoza

- (1990): "Caracteres industriales del Epipaleolítico y Neolítico en Aragón: su referencia a los yacimientos levantinos": Aragón/Litoral Mediterráneo: Intercambios culturales durante la Prehistoria. Ponencias y Comunicaciones. Instituto Fernando el Católico. Zaragoza: $177-200$

Bernabeu, J.; Aura, J.E. y Badal, E. (1993): Al oeste del Edén. Las primeras sociedades agrícolas en la Europa mediterránea. Síntesis. Madrid.

BOËDA, E. (1994): Le concept levallois: variabilité des méthodes. Monographie du CRA 9, CNRS. Paris.

Cacho, C.; Fumanal, M.P.; LóPez, P.; Pérez Ripoll, M.; Martínez Valle, R.; Uzquiano, P.; Arnanz, A.; SánChez Marco, A.; Sevillla, P.; Morales, A.; Roselló, E.; Garralda, M.D. y García Carrillo, M. (1995): "El Tossal de la Roca (Vall d'Alcalà, Alicante). Reconstrucción paleoambiental y cultural de la transición del Tardiglaciar al Holoceno Inicial”. Recerques del Museu d'Alcoi, 4: 11-102.

Carbonell, E. (ed.) (1985): Sota Palou, Campdevànol: un centre d'intervenció prehistòrica postglaciar a l'aire lliure. Centre d'Investigaciones Arqueològiques de la Diputació de Girona, sèrie monográfica 5, Girona.

Cebriá, A.; Fullola, J.M. a ; García-Argüelles, P.; GraCIA, V. y Millán, M. (1981): “Avance al estudio de los asentamientos con cerámica del Filador (Margalef de Montsant, Priorat, Tarragona)". Saguntum, 16: 37-62.

DOMÈNECH FAUS, E. (1991): "Aportaciones al Epipaleolítico del norte de la provincia deAlicante". Alberri, 3: 69-90.

- (1995): "Modèles d'habitat et systèmes de production lithique dans l'épipaleolithique de la partie centro-septentrionale de la province d'Alicante (Espagne)".
L'Europe des derniers chasseurs, 5 e Colloque International UISPP (18-23 septembre 1995). Éditions du CTHS. Paris: 101-107.

- (1998): "Los sistemas de producción lítica del Paleolítico superior final y Epipaleolítico en la vertiente mediterránea occidental. Tres ejemplos claves: la Grotte Gazel (Sallèles-Cabardès, Aude), Cova Matutano (Vilafamés Castelló) y Abric del Filador (Margalef de Montsant, Tarragona)". Pyrenae, 29: 9-45.

- (1999): Sistemas de producción líticos de la transición Paleolítico Superior Final-Epipaleolítico en la vertiente mediterránea occidental (Llenguadoc-Rosselló, Catalunya y Pais Valencià). Universitat de Barcelona, Col.lecció de Tesis Doctorals Microfitxades, 3547. Barcelona.

FORTEA, J. (1973): Los complejos microlaminares y geométricos del Epipaleolítico mediterráneo español. Memoria, 4 del Seminario Ediciones Prehistoria y Arqueología, Universidad de Salamanca. Salamanca.

Fullola, J.M. a y GarcíA-ArgüElles, P. (1996): "La formation des complexes mésolithiques de la Péninsule Ibérique". En Stephan K. Kozlowski y Carlo Tozzi (eds.): The Mesolithic, 7. The Colloquia of the XIII International Congress of Prehistoric and Protohistoric Sciences, Colloquium XIII, Forlì (Italia) 8-14 September 1996. 9-15. Forli.

García-Argüelles, P.; Adserias, M.; Bartrolí, R.; BergaDÀ, M.; Cebriá, A.; Doce, R.; Fullola, J.M. .; NadAl, J.M; RibÉ, G.; Rodón, T. y VIÑAs, R. (1992): "Síntesis de los primeros resultados del programa sobre el Epipaleolítico en la Cataluña central meridional".Aragón/ Litoral Mediterráneo: Intercambios culturales durante la Prehistoria. Ponencias y Comunicaciones. Instituto Fernando el Católico. Zaragoza: 269-284.

JuAn Cabanilles, J. (1990): "Substrat Épipaleolithique et Néolithisation en Espagne: apport des industries lithiques à l'identification des traditions culturelles". E.R.A.U.L.,39. Rubane \& Cardial, Actes du Colloque de Liege (1988): 417-435.

- (1992): "La neolitización de la Vertiente Mediterránea peninsular. Modelos y problemas".Aragón/Litoral Mediterráneo. Intercambios culturales durante la Prehistoria. Ponencias y Comunicaciones. Instituto Fernado el Católico. Zaragoza: 255-268.

LEROI-GourHAN, A. (1964): Le geste et la parole I. Technique et langage. Albin Michel. Paris.

Pelegrín, J. (1995): Technologie lithique: le Châtelperronien de Roc-de-Combe (Lot) et de la Côte (Dordogne). Cahiers du Quaternaire, 20. CNRS. Paris

Rodríguez, A. e YLl, R. (1991): "Materias primas y cadenas operativas en el yacimiento epipaleolítico del Roc del Migdia (Vilanova de Sau, Barcelona)". En R. Mora, X. Terradas, A. Parpal y C. Plana (eds.): Tecnología y Cadenas operativas. Reunión Internacional. Treballs d'Arqueología, 1, Universitat Autonoma de Barcelona (Bellaterra), Barcelona: 73-82. 
TERRADAS, X. (1995): Las estrategias de gestión de los recursos líticos del prepirineo catalán en el IX milenio B.P.: el asentamiento prehistórico de la Font del Ros (Berga, Barcelona)". Treballs d'Arqueología, 3. Universitat Autonoma de Barcelona. Barcelona.
TIXIER, J. (1991): “Cogitations non conclusives". En: 25 d'études technologiques en Prehistoire. Xième Rencontres Internationales d'Archéologie et d'Histoire d'Antibes. Editions APDCA. Juan-les-Pins: 391-394.

T. P., 57, n. $^{\circ} 1,2000$ 